reduced bone, cartilage destruction $(p<0.001)$ and less new bone formation $(p<0.001)$ in peripheral joints. Comparably, therapeutic treatment with rapamycin decreased spondylitis severity ( 1 versus $2.8 ; \mathrm{p}<0.05$ ) and arthritis severity (4 versus $8.8 ; \mathrm{p}<0.05)$ compared to controls. In the rapamycin treatment group, IL-17A $(\mathrm{p}<0.05)$ and $I L-17 F \quad(\mathrm{p}<0.05)$ mRNA were decreased in the MCP joints.

Conclusions mTOR blockade decreases IL-17A and TNF production in vitro in Spa PBMCs and reduces osteoblastic differentiation of human SpA FLS. In the M. tub induced HLA-B27 transgenic rat model of $\mathrm{SpA}, \mathrm{mTOR}$ blockade reduces arthritis and spondylitis development and severity by targeting inflammation and bone remodeling.

Disclosure of Interest None declared.

\section{P127 \\ LUNG DISEASE IN SYSTEMIC JIA: AN EMERGING PROBLEM LINKED WITH YOUNG AGE AND ANTI-IL-1/IL-6}

${ }^{1} \mathrm{G}$ Chen*, ${ }^{1} \mathrm{~V}$ Saper, ${ }^{2} \mathrm{G}$ Deutsch, ${ }^{3} \mathrm{RP}$ Guillerman, ${ }^{1} \mathrm{~K}$ Jagadeesh, ${ }^{4} \mathrm{G}$ Schulert, ${ }^{5} \mathrm{~S}$ Canna, ${ }^{1} \mathrm{Y}$ Lu, ${ }^{1} \mathrm{~J}$ Birgmeier, ${ }^{1} \mathrm{~A}$ Leung, ${ }^{4} \mathrm{~A}$ Grom, ${ }^{1} \mathrm{G}$ Bejerano, ${ }^{1} \mathrm{M}$ Davis, ${ }^{1} \mathrm{P}$ Khatri, ${ }^{1} \mathrm{E}$ Mellins, on behalf of Consortium of sJIA-LD Case Reporters. 'Stanford University, Palo Alto; ${ }^{2}$ Seattle Children's Hospital, Seattle; ${ }^{3}$ Texas Children's Hospital, Houston; ${ }^{4}$ Cincinnati Children's Hospital, Cincinnati, ${ }^{5}$ Children's Hospital of Pittsburgh, Pittsburgh, USA

\subsection{6/annrheumdis-2018-EWRR2019.115}

Career situation of first and presenting author Post-doctoral fellow.

Introduction The advent of anti-IL-1/IL-6 therapies transformed the management of systemic juvenile idiopathic arthritis (sJIA), a debilitating childhood inflammatory condition. However, concurrent with practice change, pediatric rheumatologists noted an increase in sporadic cases of types of lung disease in sJIA, that were rarely seen in previous decades.

Objectives The study aims to provide an up-to-date characterization of lung disease and identify novel risk factors.

Methods We organized a multi-center retrospective study and obtained information on 61 cases of lung disease in children with sJIA or sJIA-like disease.

Results Clinical data from our cohort showed that lung disease in the setting of sJIA is associated with distinctive features, including acute clubbing (in 61\%), atypical rash (in 56\%) and an unusually high frequency of serious adverse reactions to tocilizumab (40\%, OR $=79$ compared to the control). This lung disease has unique radiological findings and lung pathology similar to pulmonary alveolar proteinosis and/or endogenous lipoid pneumonia (PAP/ELP, 64\%) often with associated pulmonary vascular changes. Whole-exome sequencing on 18 patients ruled out a novel monogenic disorder or known causes of congenital PAP. Thus, the etiology of the disease is not clear, while its mortality is high (5 years survival: $42 \%$, $25 \%-68 \%)$. When compared to a control cohort of children entered as sJIA in a patient registry, the lung disease cohort has similar overall anti-IL-1/IL-6 exposure level. However, in the anti-IL-1/IL-6 exposed subset, but not in the non-exposed, young sJIA onset age potently increased the risk of lung disease (age $<5$ years, $\mathrm{OR}=7.3$ ). The high-risk patients (early sJIA onset and exposed to anti-IL-1/IL-6) were highly enriched for PAP/ELP pathology $(89 \%, 17 / 19$ vs. low-risk: 35\%, 6/17). In addition, children with Trisomy 21 (T21) and sJIA have dramatically elevated risk for lung disease $(\mathrm{OR}=50)$. Marked serum ferritin elevation and/or profound lymphopenia occur several months prior to lung disease detection and may be early indicators of lung disease.
Conclusions Lung disease associated with anti-IL-1/anti-IL-6 therapies often presents as an alveolar and pulmonary vascular complication with high mortality in children with sJIA/sJIAlike disease. Risk is significantly increased in children with young age at sJIA onset or concurrent T21. In sJIA patients (including those with initial treatment response), emergence of atypical clinical features, rising ferritin and dropping absolute lymphocyte count should raise suspicion of this complication.

Disclosure of Interest G. Chen: None declared, V. Saper: None declared, G. Deutsch: None declared, R. P. Guillerman: None declared, K. Jagadeesh: None declared, G. Schulert: None declared, S. Canna: None declared, Y. Lu: None declared, J. Birgmeier: None declared, A. Leung: None declared, A. Grom: None declared, G. Bejerano : None declared, M. Davis: None declared, P. Khatri: None declared, E. Mellins Grant/research support from: Novartis, GlaxoSmithKline, Codexis, Inc.

\section{\begin{tabular}{l|l} 
P128 & AN ADD-ON TRAINING PROGRAM INVOLVING
\end{tabular} BREATHING EXERCISES, COLD EXPOSURE, AND MEDITATION ATTENUATES INFLAMMATION AND DISEASE ACTIVITY IN AXIAL SPONDYLOARTHRITIS - A PROOF OF CONCEPT TRIAL}

${ }^{1} \mathrm{H}$ De Jong, ${ }^{1} \mathrm{G}$ Buijze, ${ }^{2} \mathrm{M}$ Kox, ${ }^{1} \mathrm{M}$ van de Sande ${ }^{*},{ }^{1,3} \mathrm{D}$ J van Schaardenburg, ${ }^{1} \mathrm{R}$ van Vugt, ${ }^{2,4} \mathrm{C}$ Popa, ${ }^{2} \mathrm{P}$ Picckers, ${ }^{1} \mathrm{D}$ Baeten. 'Amsterdam UMC, Amsterdam; ${ }^{2}$ Radboud UMC, Nijmegen; ${ }^{3}$ Reade, Amsterdam; ${ }^{4}$ Bernhoven, Uden, Netherlands

\subsection{6/annrheumdis-2018-EWRR2019.116}

Career situation of first and presenting author Student for a master or a $\mathrm{PhD}$.

Introduction Previous research in healthy individuals showed that the innate immune response can be voluntarily modulated by a training program involving breathing exercises, exposure to cold and meditation ${ }^{1}$. It is unknown whether the same intervention could potentially lead to suppression of inflammation and whether it can safely be applied in patients with chronic inflammatory diseases.

Objectives The objective of this study was to assess safety and anti-inflammatory effects of an add-on training program comprising breathing exercises, cold exposure and meditation in patients with axial spondyloarthritis.

Methods This was an open-label randomised one-way crossover proof-of-concept trial. Participants were randomised to either the early or late intervention group. The intervention consisted of an 8 weeks training program comprising cold exposure, meditation and breathing exercises.

Results There was no significant difference in adverse events between groups, with one serious adverse event occurring 8 weeks after end of the intervention and judged 'unrelated'. After 8 weeks there was a significant decline of the hs-CRP in the intervention group (median [interquartile range] from 10.2 [6.5-17.1] to $6.0[3.9-15.6] \mathrm{mg} / \mathrm{L} ; \mathrm{p}=0.02)$, but not in the control group (from $8.7[5.2-17.2]$ to 13.2 [7.9-20.1] $\mathrm{mg} / \mathrm{L}$; $\mathrm{p}=0.70)$. A similar trend was seen for serum calprotectin ( $p=0.07$ in intervention versus $p=0.31$ in control), but not for ESR. In addition, ASDAS-CRP $(p=0.02)$, BASDAI $(\mathrm{p}=0.04)$, SF-36 physical component score $(\mathrm{p}=0.01)$, EQ-5D $(p=0.049)$, and EQ-5D VAS $(p=0.02)$ decreased significantly in the intervention group, but not the control group.

Conclusions In the present trial we show that the add-on training program involving breathing exercises, cold exposure, and meditation can safely be applied in patients with axial 